\title{
Personalized Recommendation Service System in E-Learning Using Web Intelligence
}

\author{
Xinye $\mathrm{Li}^{1}$, Qi Luo ${ }^{2,3}$, and Jinsha Yuan ${ }^{1}$ \\ ${ }^{1}$ Department of Electronic and Communication Engineering,North China Electric \\ Power University, Baoding 071003, China \\ ${ }^{2}$ Information Engineering school, \\ Wuhan University of Science and Technology and Zhongnan Branch, \\ Wuhan 430223, China \\ ${ }^{3}$ College of Engineering and Technology, Southwest University, \\ Chongqing 400715, China \\ yljh654@sina.com, ccnu_luo2008@yahoo.com.cn
}

\begin{abstract}
Personalized education is a very hot topic in distant research. To realize personalized E-learning, a personalized recommendation service system was proposed and realized. Web technologies of personality and adaptive filtering were applied in the system. The structure of system, workflow and key technologies of realizing feature selection module, user interest module, personalized teaching resources filtering module were introduced in the paper.
\end{abstract}

Keywords: E-Learning, Data Mining, Web Intelligence, Personality.

\section{Introduction}

Nowadays, personalized education is a very hot topic in E-learning research. The importance of E-Learning has been transferred from how to solve the limitation of space-time problem in traditional teaching to build up the personalized learning environment, and offer a kind of personalized service based on modern pedagogy and psychology theories[1]. The learners are different in age level, sex, and social role, their culture, education background, attention, interest hobby are also exist a great difference. Giving corresponding learning contents and tactics to realize teaching according to learners' needs is very difficult [2]. The investigation indicates that personalized service system for E-Learning is imperfect. The validity and accuracy of providing information are low, which makes learners feel unconfident and suspicious in E-learning. If E-learning wants to attract more learners, the idea of personalized design should be needed. It means that the personalized knowledge and information service should be provided according to learners' needs. The key of personalized design is how to recommend teaching resources based on their interests by using web intelligence technology.

Web intelligence has been recognized as a new direction for scientific research and development to explore the fundamental roles as well as practical impacts of artificial intelligence and advanced information technology on the next generation of 
web-empowered products, systems, services, and activities. It is one of the most important as well as promising IT research fields in the era of web and agent intelligence [3].

Basing on it, web technologies of personality and adaptive filtering are applied in the paper [4], while the model of personalized E-learning recommendation service system (PERSS) is proposed.

\section{System Structure}

Personalized E-learning recommendation service system (PERSS) is showed in Fig.1

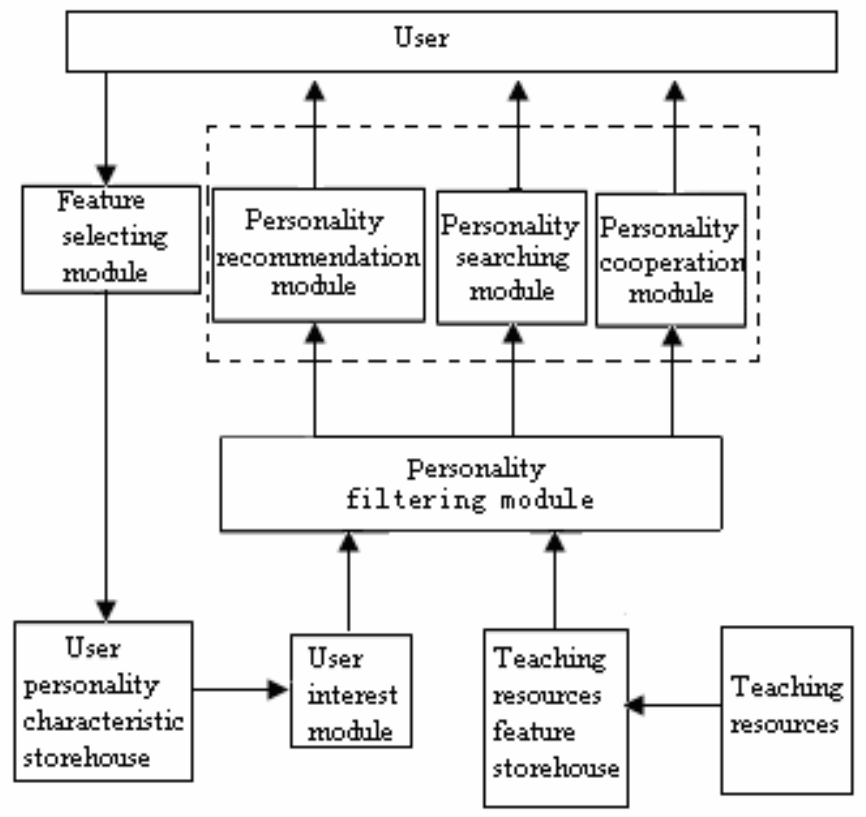

Fig. 1. Personalized recommendation service system

The main function of PERSS is to help users to find out teaching resources quickly and accurately according to their interests.

Feature selecting module selects users' interested teaching resources information according to browsing and feedback of teaching resources. Then, the information is stored in user personality characteristics storehouse that is tracked and updated promptly.

User personality characteristics storehouse saves user personality information such as name, age, occupation, purchasing interest, hobby and so on. It tracks users' interests dynamically. The key words of user personality characteristics are selected and recorded; it can also provide user characteristics for constructing the module of user interest model. 
The module of user interest model selects key words from user personality characteristics storehouse and constructs user interest model.

The filtering module of personality teaching resources filters teaching resource according to user model.

Personality recommendation module realizes automatic recommendation and request recommendation. Request recommendation is realized by calling the management module of teaching resources and the filtering module of teaching resources.

Personality searching module accepts user requests. Personality searching result is obtained through filtering module.

Personality cooperation module makes user get help immediately and accurately when they have problems of learning.

The work process of model is as follows: firstly, user interest feature information of teaching resources is selected by feature selecting module. The information is saved in user personality characteristics storehouse. Personality characteristics storehouse is tracked and updated promptly. Then, user interest model is constructed by user personality characteristics information. Furthermore, teaching resources are filtered by filtering module of personality teaching resources according to user model. Finally, personality searching service, personality recommendation service and personality cooperation service are realized by the module of personality searching, personality recommendation and personality cooperation, which according to the filtering results.

\section{Key Technologies}

\subsection{User Interest Model}

The process of establishing user interest model is the process of knowledge acquisition. There are two ways of knowledge acquisition such as user obvious feedback and user hiding feedback [5].

In order to fully utilize the advantages of the two ways, user interest initial model is established according to user register information and user interest model is updated according to web server log. A web server log records user's IP address, visiting time, visiting page, visiting way, requested the document URL ,HTTP version, return codes, transmission byte, protocol, wrong codes and so on.

\subsubsection{Establishing User Interest Model}

User initial interest model is established according to user register information. User register information is submitted to the system server though Web inputting. When user register, the Web may let the user reply some questions such as research orientation, interests and so on. Different people' answers are formed different personalized vectors which express user interests.

Supposed user interest model vector is $Q_{i}$, the number of interest feature item is n, and the interest number is $\mathrm{m}$, then user initial interest model $\mathrm{U}$ may be represented as a vector matrix $U=\left(Q_{1}, Q_{2}, \cdots Q_{m}\right)$, interest vectors $Q_{i}=\left(q_{i 1}, q_{i 2}, \cdots q_{i n}\right)$. 


\subsubsection{Updating User Interest Model}

Because user interest is variable, user interest model is updated through observing user's behavior in web server log. When website has been visited one time, log database increases corresponding records; these records are arranged from visiting time. Mining these log documents may discover what the user has browsed and how long the user has browsed the page.

Generally speaking, the user may visit interesting pages, if the user browses the page repeatedly, then it shows that user is interested in this page. Therefore, mining this page could find out user interests and interest degree. $Q_{1}, Q_{2}, \cdots Q_{m}$ represent all interest vectors in user interest model, supposed vectors $X_{1}, X_{2} \cdots X_{d}$ represent all content pages that user has visited. If page $X_{j}$ approaches to user interest $Q_{i}$ namely, $Q_{i} X_{j} /\left|X_{j}\right|$ is bigger than the thresholds a, then page $X_{j}$ vector is add to the user interest $Q_{i}$

$$
Q_{i}=Q_{i}+\frac{m^{2} X_{j} X J(j) T(j)}{\sum_{k=1}^{m} X J(k) \sum_{k=1}^{m} T(k)}
$$

$X J(j)$ represents the frequency of visiting the page, $T(j)$ represents the time of visiting the page, $j=1,2, \cdots m$.

If page $X_{j}$ does not approach to user's any interest $Q_{i}$, then $X_{j}$ is regarded as user new interest. But the category of user interest increases massively, then we must establish the thresholds $b$, if the mold of user interest vector is smaller than $b$, pushing correlative pages don't consider this interest.

Page browsing also is random and regular. If user is interested in the page, then the user will consume more time when browsing it, simultaneously also visit this page repeatedly. The following formula 2 is used to calculate user interest degree.

$$
F=\left(\frac{n}{N}+\frac{t}{T}\right) \frac{k}{K}
$$

Where $\mathrm{F}$ is interest degree of visiting the node, $\mathrm{n}$ is the number of times in visiting the node, $\mathrm{N}$ is the total number of times in this visit, $\mathrm{t}$ is the consuming time in visiting the node, $\mathrm{T}$ is the total time in visiting website, $\mathrm{k}$ is number of node in this visit, $\mathrm{K}$ is the total number of node in visiting website.

\subsection{Filtering Module for Teaching Resources}

Adaptive filtering algorithm for teaching resources based on vector space model is composed of three steps such as training, adaptive filtering and modifying thresholds adaptively.

Step1: training phase. According to assigned training texts, initial filtering profiles and thresholds are generated [6].

Step2: adaptive filtering phase. For each text in teaching resources, we judge whether it is related with initial filtering profiles or not. 
Step3: modifying thresholds adaptively. Users' feedback is utilized to modify the profiles and thresholds adaptively.

\subsubsection{Training}

Fig. 2 is the flow of training algorithm. Firstly, topics are converted to vector forms. Then feature vectors are separately obtained from user interest model, positive samples and pseudo positive samples. So, initial profiles vectors are composed of positive samples vectors, pseudo positive samples vectors, user interest vectors and topic vectors. Then the similarity between initial profiles vectors and all training samples is computed, so we can obtain the best initial thresholds.

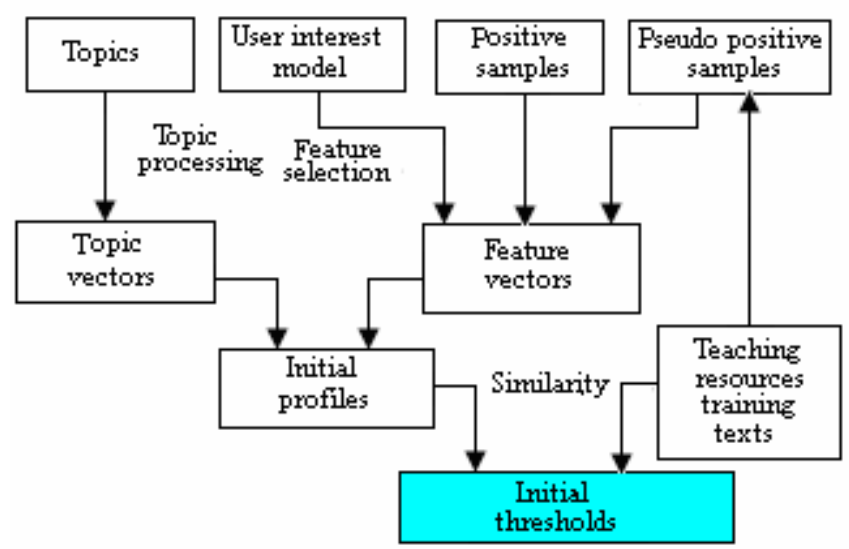

Fig. 2. The flow of training algorithm

\subsubsection{Adaptive Filtering}

After Initial filtering profiles are set up and initial thresholds are selected, the process of filtering is to modify filtering profiles and thresholds adaptively. It is just the process of machine learning that improves filtering performance. Fig. 3 is the flow of adaptive filtering algorithm. For each text in the teaching resources, the similarity between it and profiles vectors is computed. If the similarity is bigger than the thresholds, then it is regarded as the related texts. Whether the texts are really related to the topics or not is judged by users. Profiles and thresholds are modified according to user' results.

\subsubsection{Modifying Thresholds Adaptively}

The proportion of related texts in teaching resources is very low, so it is necessary to modify thresholds adaptively. The goal of elevating thresholds is to filter less positive samples and increase precision. The goal of lowering thresholds is to filter more positive samples. Probability density distribution is proposed in the paper. If probability density distribution of positive samples currently surpasses the expectation of distribution scope, then the thresholds would be elevated. Otherwise, thresholds would be lowed. 


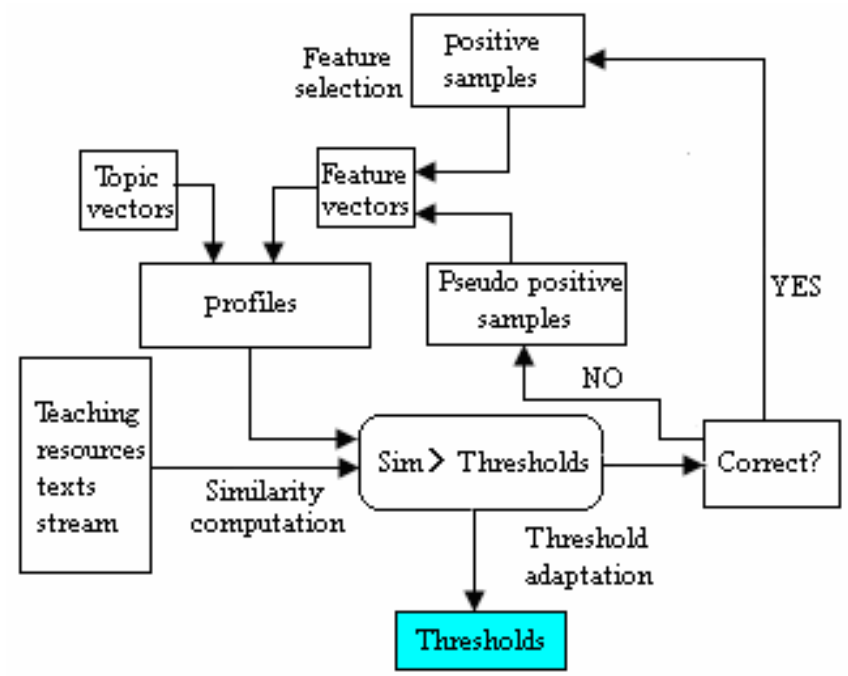

Fig. 3. The flow of training algorithm

Definition 1. $\mathrm{n}$ is the serial number of filtering texts in teaching resources. $\mathrm{D}$ is the expectation of positive samples distribution density.

Definition 2. S (n) is the number of total documents which the documents' serial number is up to $n$.

Definition 3. SR (n) is positive samples which the documents' serial number is up to $\mathrm{n}$ Definition 4. O (n) is thresholds which the documents' serial number is up to n.

Definition 5. DR $\left(\mathrm{n}_{t}, \mathrm{n}_{t+1}\right)$ is probability density distributing of previous thresholds.

$$
D R\left(n_{t}, n_{t+1}\right)=\frac{S R(t+1)-S R(t)}{S(t+1)-S(t)}
$$

Thresholds adjusting algorithm is as follows:

(1) If $\mathrm{DR}\left(\mathrm{n}_{t}, \mathrm{n}_{t+1}\right)>\max (\mathrm{D}, 0.3)$ and $\mathrm{SR}(\mathrm{n})<0.3 \mathrm{~S}(\mathrm{n})$, then $\mathrm{O}(\mathrm{n}+1)=\mathrm{O}(\mathrm{n}) * 1.3$.If precision is lower excessively and the number of filtering texts is not too few, then thresholds are elevated rapidly.

(2) If DR $\left(\mathrm{n}_{t}, \mathrm{n}_{t+1}\right)>\mathrm{D}$, then $\mathrm{O}(\mathrm{n}+1)=\mathrm{O}(\mathrm{n}) * 1.2$. If the number of filtering texts is more than what we need, then thresholds are elevated.

(3) If DR $\left(\mathrm{n}_{t}, \mathrm{n}_{t+1}\right)<D$, then $\mathrm{O}(\mathrm{n}+1)=\mathrm{O}(\mathrm{n}) * 0.8$.If the number of filtering texts is less than what we need, then thresholds are lowered.

\section{Conclusion}

Based on the above research, the author constructs a system website recommending personalized courses service .In order to obtain the contrast experimental results, 


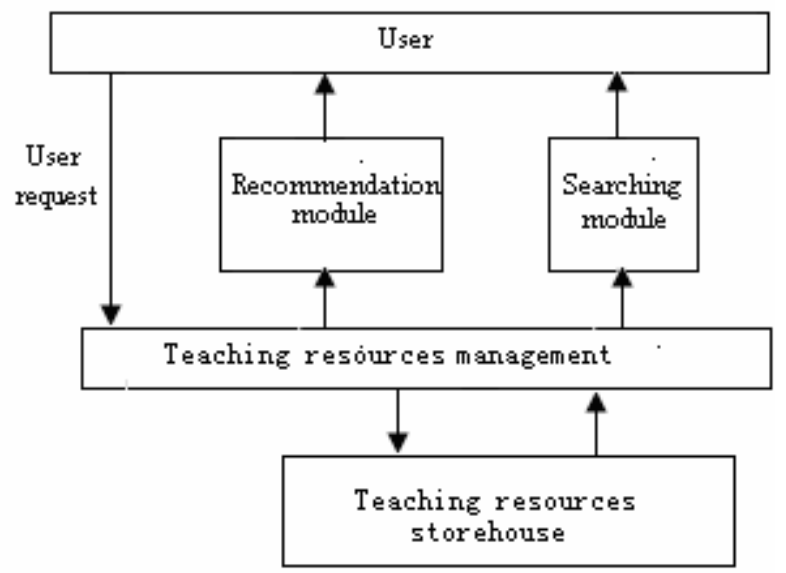

Fig. 4. Contrast system

feature selection module, user interest module, personalized teaching resources filtering module are removed from PERSS, and the contrast system is formed. The contrast system is Fig.4.

Evaluation metrics as follows [7]:

$$
\begin{gathered}
\text { Pr ecision }=\frac{\text { number of inf ormation correctly filtered }}{\text { number of inf ormation filtered }} \\
\operatorname{Recall}=\frac{\text { number of inf ormation correctly filtered }}{\text { number of inf } \text { ormation }} \\
F_{\beta}=\frac{\left(\beta^{2}+1\right) \times \text { Precision } \times \operatorname{Re} \text { call }}{\text { Precision }+\operatorname{Re} \text { call }}
\end{gathered}
$$

There are 150 people register in this system. 150 personality characteristics storehouse are established. The interest group is 41; there are 341 texts in teaching resources. 12 texts that have the biggest correlation in teaching resources are provided to the corresponding user. Through the experiment, table 1 shows the results:

Table 1. The experiment results

\begin{tabular}{|c|c|c|c|}
\hline System & Avg.Precision & Avg.Recall & $F_{\beta}$ \\
\hline PERSS & 0.34 & 0.23 & 0.28 \\
\hline $\begin{array}{c}\text { Contrast } \\
\text { System }\end{array}$ & 0.13 & 0.11 & 0.12 \\
\hline
\end{tabular}

From the table1, we discover that the filtering average precision of PERSS is higher than the contrast system. 
In summary, the model of personalized E-learning recommendation service system using web intelligence is proposed and realized in the paper. Web intelligence of adaptive filtering algorithm is also used in personalized E-learning recommendation service system.

\section{References}

1. Luo Q.: Research on Application of Association Rule Mining Algorithm in Learning Community. Proceedings of CAAI-11, Wuhan, 2005, 1458-1462.

2. Yanwen W. and Zhonghong W..:Knowledge Adaptive Presentation Strategy in E-Learning. Proceedings of Second International Conference on Knowledge Economy and Development of Science and Technology, Beijing, 2004, pp.6-9.

3. Feng Shicong.: Research of personalization technologies based on web mining.Computer Engineering and Design.No.1, 2004.

4. Yu Li, Liu Lu.:Comparison and Analysis on E-Commence Recommendation Method in china. System Engineering Theory and Application, 2004(8), 96-98.

5. Nie Xin.:Take about the Digital Individualized Information Service of Library. Information Science Journal, vol.23, 2005, 1-5.

6. Robertson S, Hull DA.:The TREC-9 filtering track final report. Proceedings of the 9th Text Retrieval Conference. Gaithersburg, 2001, 25-40.

7. $\mathrm{Hu}$ Tian, Xia Yingju and Huang Xuanjing. :A Web-based Chinese Information Filtering System Base on VSM. Computer engineering, Beijing, vol.29, 2003, 25-27. 